\title{
Flow Control Based on Constant Pressure Microfluidic Pump System
}

\author{
Lu Limin \\ State Key Laboratory of Bioelectronics \\ School of Biological Science and Medical Engineering, \\ Southeast University \\ Nanjing, PR China \\ 220131591@seu.edu.cn
}

\section{Ke Ruichang}

State Key Laboratory of Bioelectronics

School of Biological Science and Medical Engineering,

Southeast University,

Nanjing, PR China

220131626@seu.edu.cn

\author{
Zeng Shenglan \\ State Key Laboratory of Bioelectronics \\ School of Biological Science and Medical Engineering, \\ Southeast University \\ Nanjing, PR China \\ 220143725@seu.edu.c

\section{Liu Quanjun} \\ State Key Laboratory of Bioelectronics \\ School of Biological Science and Medical Engineering, \\ Southeast University \\ Nanjing, PR China \\ lqj@seu.edu.cn
}

\begin{abstract}
Microfluidic driving and controlling techniques are fundamental and important in microfluidic applications. Traditional reagent driving systems of microfluidic chips such as syringe pump and piezoelectric actuator have their inherent shortcomings. Syringe pumpis easy to deformation and screw motion is unstable mayaffect flow rate. Whilethe flow range of piezoelectric actuator is too narrow.To solve these problems we establisheda constant pressure pump by maintaining stable pressure in a cylinder through the switch of electromagnetic valves, and then liquidin the pressurized reservoiris driven out. A simple PID algorithm is utilized to balance cylinder pressure,while flow rate is measured by a flow sensor. This setupcontains a wireless module that can communicate with a PC through Wi-Fi. We use 50uminner diameter flow resistors with different lengths to verify the relationship among flow rate, flow resistor length and pressure drop.The pump could generate a constant pressure in cylinder and the fluctuation of liquid flow is small.This system can be used as injection system of microfluidic chips and it can also be applied to other biochemical reaction. Operation is easy and users can control the system at a distance.
\end{abstract}

Keywords-injection system;constant pressure;flow rate; wireless communication; flow resistors

\section{INTRODUCTION}

Microfluidic technology has numerous applications such as cell analysis, cell culture, DNA analysis, drug research and immunoassay and so on. Reagent sampling, dilution, injection, reaction, separation, detection and analysis can be completed in the microfluidic analysis system by manipulation of the fluid inside microchannel[1]. Manipulation of fluid is the basis of microfluidic analysis system, therefore fluid driving device with small volume and small injection port is necessary to achieve the control of fluid[2,3]. The core technique of microfluidic driving device is its injection mode and the corresponding driving mode. Using different theories people have invented various kinds of microfluidic driving and control technology that taking advantage of pressure, electricity, heat, surface tension, centrifugal force and so on[4-6].

Recently there are three kinds of commonly used methods which utilize pressure: syringe pump, piezoelectric actuator and pneumatic pump.

\section{A. Syringe pump}

Syringe pump consists of stepper motor and its drive, screw rod and matched screw nut that can move reciprocating, bracket, etc. SCM system sends control pulses to the stepper motor then stepper motor drive the screw rod and transform rotation movement to linear motion therefore push the syringe piston for fluid injection. Syringe pump implement fluid transmission without pulse and precise control of fluid[7]. There are several common disadvantages of syringe pump such as quick wear of the injection unit and residual air is not easy to eliminate.

\section{B. Piezoelectric actuator}

Piezoelectric actuator is a kind of micropump that utilizes inverse piezoelectric effect of piezoelectric materials under a periodic electric field to drive pump diaphragm move to-and-fro. Piezoelectric actuator has a simple structure, high jet frequency, quick response, while only a small scale of liquid viscosity can be distributed.

\section{Pneumatic pump}

Pressure pump drive liquid flow by connecting an external independent pressure source to inlet ports. The liquid flow is partially affected by the hydraulic-head difference that between the gas-inlet and drain-outlet of reservoirs. The compressibility of air and the response time of magnetic valves could influence the accurate control of pressure[8-10]. Constant pressure in the cylinder should be maintained by measuring real-time 
pressure and precisely regulating the solenoid valves, so we can accurately control the flow of fluid.

In summary, there are various innovative approaches attempted to precisely control the injection of fluids. Here we establish a constant pressure microfluidic pump providing a stable pressure to drive reagent into channels of microfluidic chips. With an external nitrogen gas source connected to four solenoid vales, the pressure in the cylinder can be regulated by the switch on and off of solenoid vales whose actions are controlled by the SCM system. Users can set pressure and flow values by operating PC interface while the instructions are send to the SCM through Wi-Fi communication. The computer can receive data from $\mathrm{SCM}$ in real time and draw pressure and flow curves at the same time.

\section{SYSTEM DESIGN}

\section{A. Whole design of this system}

The overall constant pressure microfluidic pump system consists of three subsystems: (1) a constant gas pressure generation and control subsystem including related electronic printed circuit boards, electromagnetic valves and a Wi-Fi communication module (pneumatic subsystem); (2) a $510 \mathrm{~mm}$ long liquid pipe with $250 \mu \mathrm{m}$ inner diameter, corresponding fittings and various hydraulic resistors with different lengths (micro-channels); (3) PC control system utilizing a laptop with wireless module. The overall framework is shown in Fig.1.

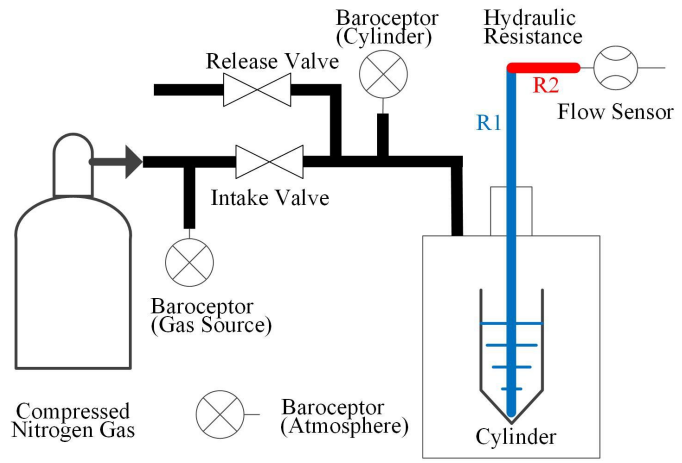

Figure 1. Overall framework

\section{B. Pneumatic subsystem}

The pneumatic subsystem is designed to generate a constant pressure in the cylinder. The external nitrogen gas source provides a continuous pressure for this system. These three barometric sensors detect atmosphere pressure, source pressure and cylinder pressure respectively. One two-way normally-closed electromagnetic valve is responsible for the gas input while the other two-way electromagnetic valve is used for release gas. A MCU sends electrical pulses to these two valves to control their switching time, air inflow of cylinder is affected by switching time of valves and pressure difference between gas source and the cylinder. To precisely and rapidly control the cylinder pressure we employ a simple PID algorithm to regulate switching time of valves.

The whole hardware circuit diagram of this systemis showed in Fig. 2 and the software flow diagram is showed in Fig.3, if the set pressure or set flow value is greater than current value, then we open the inlet valve to intake air, while on the contrary we open the vent valve to release air.

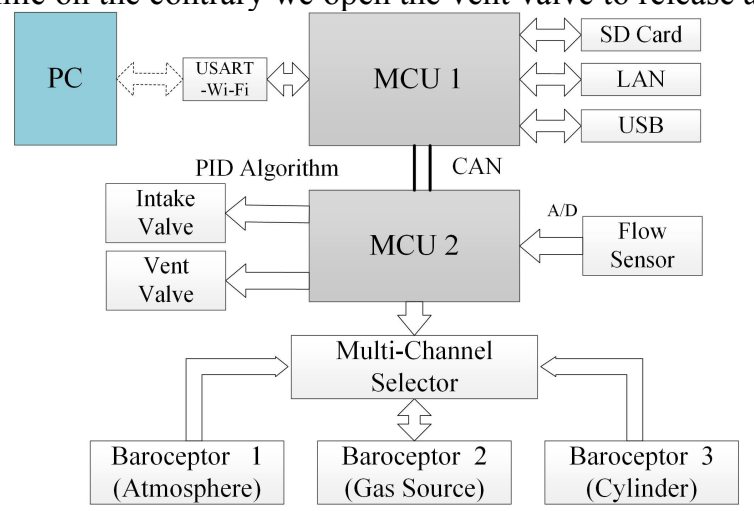

Figure 2. Hardware circuit diagram

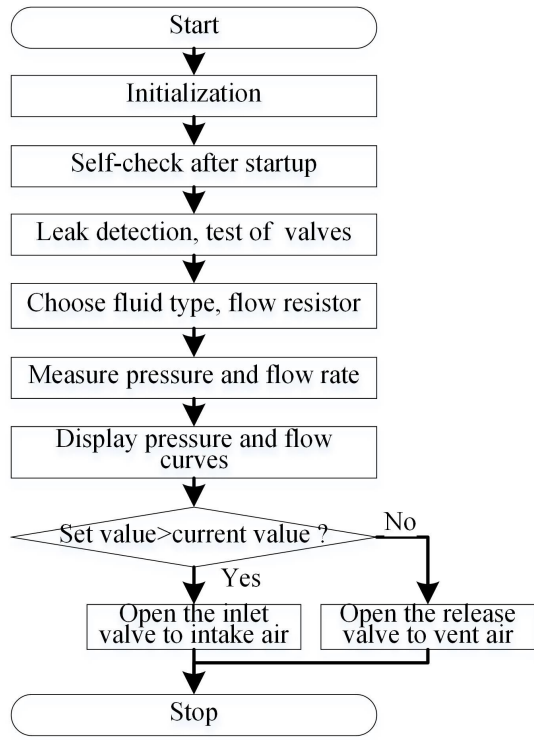

Figure 3. System software flow diagram.

\section{Micro-channels}

The constant pressure drive reagents in the reservoir flow into a channel with $250 \mu \mathrm{m}$ inner diameter. Before flowing into the flow sensor, the reagent first flow through a length of flow resistance with different lengths. The flow sensor measure flow rate in real time and convey analog electrical signals to $\mathrm{AD}$ port of the single chip microcomputer, then the AD module convert these analog signals to digital signals. We can calculate the flow rate value using a special formula.

In addition to regulate the flow rate by changing pressure of the cylinder, we can also utilize various flow resistances to change the flow rate.

\section{PC software}

PC software with a graphical user interface was written to control the whole system, users can set pressure and flow values using this software. This software has three basic functions: (1) send instructions to SCM; (2) receive pressure and flow data from SCM and display corresponding curves; (3) store pressure and flow data and save operating information of users. The advanced features 
consist of account management, database access, set the working time, scan experiment results such as Excel sheets and pictures, cooperative work and so on.

\section{EXPERIMENT}

\section{A. PID Algorithm}

We utilize a simple PID algorithm to generate a constant pressure by switching on and off the solenoid valves. The process of PID control is shown in Fig.4.

All baroceptors detect pressure values of the cylinder in real-time. If current pressure value in cylinder is not equal to target value, we will calculate the opening time of valves. $K_{\mathrm{P}}$ and $K_{\mathrm{I}}$ represent proportion coefficient and integral coefficient respectively. These parameters are dynamical changed in the process of working.

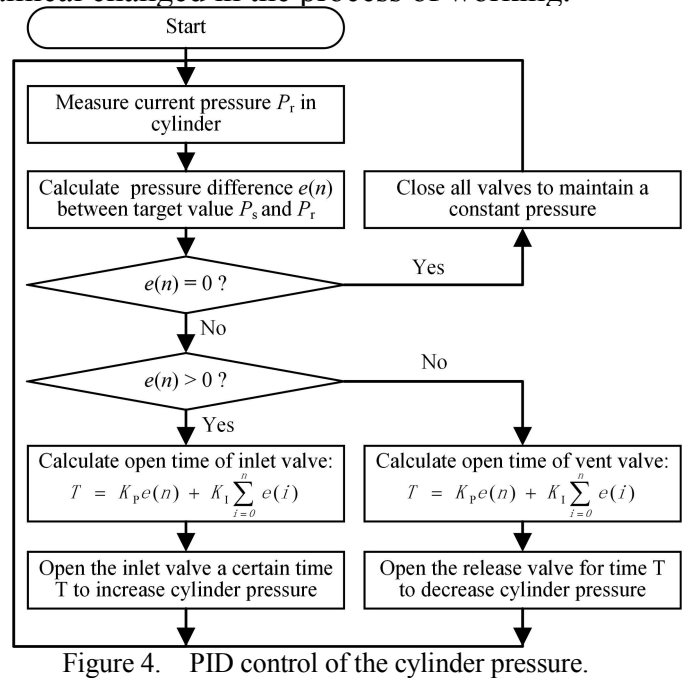

\section{B. Hagen-Poiseuille's Law}

The volumetric flow rate $Q\left[\mathrm{~m}^{3} \mathrm{~s}^{-1}\right]$ for the constant pressure-driven fluid in the circular channel is described as below:

$$
Q=\frac{\pi R^{4}}{8 \mu}\left(-\frac{\mathrm{d} p}{\mathrm{~d} x}\right) .
$$

We assume that the pressure gradient along the channel is uniform in constant pressure-driven devices, then axial pressure drop $-\mathrm{d} p / \mathrm{d} x$ can be approximate into $\Delta p / L$ where $\Delta p$ is the pressure difference [Pa] through a finite channel length $L . \mu$ is the viscosity[Pa.s] of the liquid, here $\mu$ equals to $1.009 \mathrm{mPa}$.s because the room temperature is 20 degrees centigrade. $R$ is the radius of channel. Then the formula becomes[11]

$$
Q=\frac{\pi R^{4}}{8 \mu} \frac{\Delta p}{L}
$$

Then (2) can be rewritten as

$$
Q=\frac{\Delta p}{R_{\mathrm{H}}} .
$$

The flow resistance $R_{\mathrm{H}}$ is defined as

$$
R_{\mathrm{H}}=\frac{8 \mu L}{\pi R^{4}} \text {. }
$$

There are two kinds of pipes series-connected in this system, flow resistance of the first pipe is $R_{1}$ and the secondone is $R_{2}$. All flow resistance of pipes is shown in TABLEI.The total hydraulic resistance of these seriesconnected fluidic resistors is the sum of $R_{1}$ and $R_{2}$. The unit of flow rate is $\mu \mathrm{l} / \mathrm{min}$ and the unit of pressure is mbar in experiment and later calculation.
TABLE I. Flow Resistance of INVOLVED CHANNELS

\begin{tabular}{ccc}
\hline $\begin{array}{c}\text { Length } \\
(\mathrm{cm})\end{array}$ & $\begin{array}{c}\text { Inner Diameter } \\
(\mu \mathrm{m})\end{array}$ & $\begin{array}{c}\text { Flow Resistance } \\
\left(\mathrm{Pa}_{\mathrm{sm}}^{-3}\right)\end{array}$ \\
\hline 51 & 250 & $5.367368^{*} 10^{12}$ \\
5 & 50 & $3.288829 * 10^{14}$ \\
10 & 50 & $6.577657^{*} 10^{14}$ \\
15 & 50 & $9.866486 * 10^{14}$ \\
20 & 50 & $1.315532 * 10^{15}$ \\
25 & 50 & $1.644414 * 10^{15}$ \\
\hline
\end{tabular}

\section{RESULTS AND DISCUSSION}

To generate a stable flow rate we should first sustain a constant pressure. We use a simple PID algorithm to regulate the cylinder pressure. After users send set values to the system, a relatively stable time-varying pressure is shown in Fig.5. The fluctuation of cylinder pressure is \pm 2 mbar.

Flow rate is inversely proportional to flow resistor length for the same inner diameter $(50 \mu \mathrm{m})$ and fixed pressure drop, as shown in Fig.6. Values in the legend are absolute values. Pressure drop $\Delta p$ equals to current cylinder pressure minus the environment pressure value. Flow fluctuation is small during experiment.

Equation (3) denotes that the flow rate $Q$ is proportional to the pressure drop $\Delta p$ for a certain flow resistance $R_{\mathrm{H}}$.Fig. 7 indicates that flow rate conforms to this formula. Fig. 8 shows the ideal flow rate curve(blue curve) based on (3) and a real curve (black curve) and its fitting curve (red line) for a $10 \mathrm{~cm}$ channel.

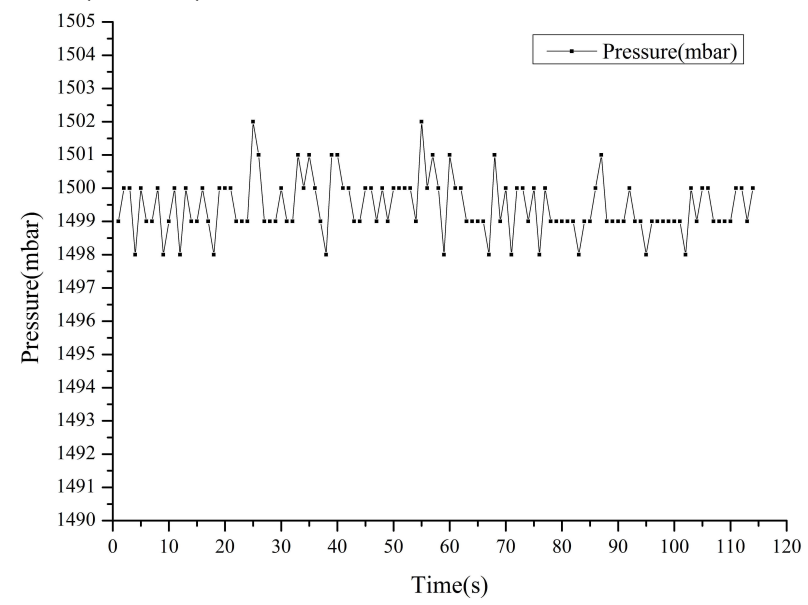

Figure 5. Cylinder pressure changes with time.

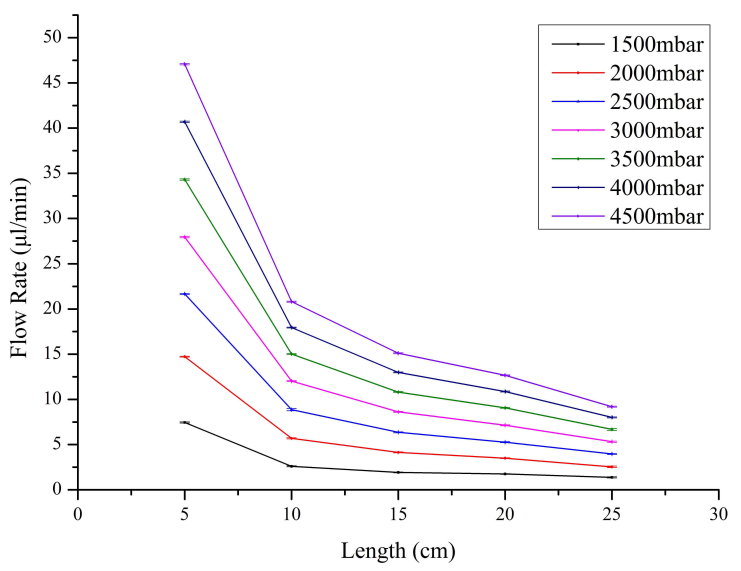

Figure 6. Flow rate varies with flow resistor length. 


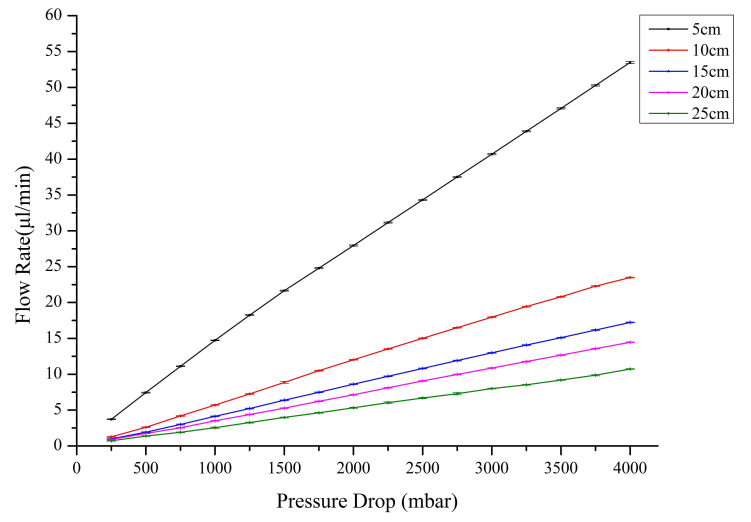

Figure 7. Flow rate varies with pressure drop.

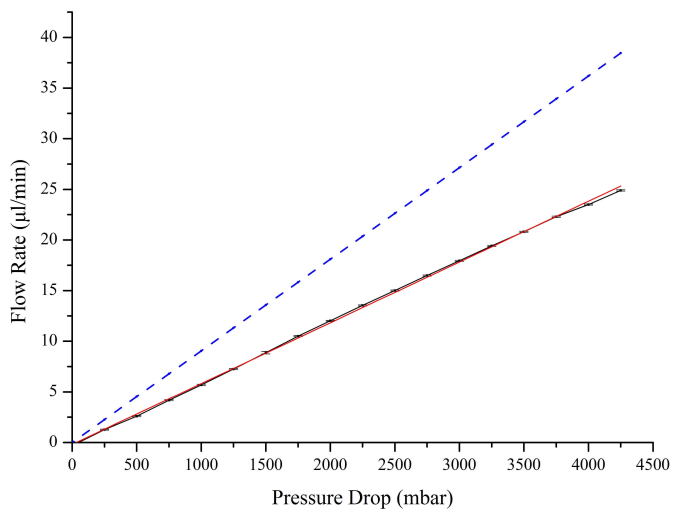

Figure 8. Ideal curve and real curve for a $10 \mathrm{~cm}$ channel.

The fitting linear equation of the flow rate of $10 \mathrm{~cm}$ channel is

$$
Q=0.006 * \Delta p-0.19147 \text {. }
$$

Here the unit of flow rate $Q$ is $\mu 1 / \mathrm{min}$ and the unit of pressure $\Delta p$ is mbar. The slope is $1 / R_{\mathrm{H}}=0.009048$ in theory, but using this system the real slope is 0.006 . Because some flow resistance of our system has not beencalculated such as the resistance of pipe joints, the real slope is not ideally conforms to the ideal slope.

\section{CONCLUSION}

In summary, we have demonstrated this constant pressure microfluidic pump system could maintain a stable pressure in the cylinder and drive reagents to flow steady. In addition to set different pressure values, we can also choose different kinds of flow resistors to regulate the flow rate. Users can configure the system conveniently according to their target flow range. This system has a wide flow rate range and it is mainly used as injection system of microfluidic chips, it can also be applied to other biochemical reaction.

\section{ACKNOWLEDGMENT}

This work was supported by National Basic Research Program of China (2011CB707600), the National Natural Science Foundation of China (61071050, 61372031), Tsinghua National Laboratory for Information Science and Technology (TNList), Cross-discipline Foundation and "the Fundamental Research Funds for the Central Universities", and the Foundation for the author of National excellent doctoral dissertation.

\section{REFERENCES}

[1] Q. Zhang, P. Zhang, Y. Su, C. Mou, T. Zhou, M. Yang, J. Xu and B. $\mathrm{Ma}$, "On-demand control of microfluidic flow via capillary-tuned solenoid microvalve suction", Lab Chip, 14, 2014, p. 4599-603.

[2] J.-S. Chen and J.-H. Jiang, "Droplet Microfluidic Technology: Mirodroplets Formation and Manipulation", Chinese Journal of Analytical Chemistry, 40, 2012, p. 1293-1300.

[3] K. Churski, M. Nowacki, P.M. Korczyk and P. Garstecki, "Simple modular systems for generation of droplets on demand", Lab Chip, 13, 2013, p. 3689-97.

[4] Z. Wu, H. Jensen, J. Gamby, X. Bai and H.H. Girault, "A flexible sample introduction method for polymer microfluidic chips using a push/pull pressure pump", Lab Chip, 4, 2004, p. 512-5.

[5] D.H. Yoon, A. Jamshaid, J. Ito, A. Nakahara, D. Tanaka, T. Akitsu, T. Sekiguchi and S. Shoji, "Active microdroplet merging by hydrodynamic flow control using a pneumatic actuator-assisted pillar structure", Lab Chip, 14, 2014, p. 3050-5.

[6] L. Clime, D. Brassard, M. Geissler and T. Veres, "Active pneumatic control of centrifugal microfluidic flows for lab-on-a-chip applications", Lab Chip, 15, 2015, p. 2400-11.

[7] X. Bai, H.J. Lee, J.S. Rossier, F. Reymond, H. Schafer, M. Wossner and H.H. Girault, "Pressure pinched injection of nanolitre volumes in planar micro-analytical devices", Lab Chip, 2, 2002, p. 45-9.

[8] M. Rhee and M.A. Burns, "Microfluidic pneumatic logic circuits and digital pneumatic microprocessors for integrated microfluidic systems", Lab Chip, 9, 2009, p. 3131-43.

[9] J.-W. Choi, S. Lee, D.-H. Lee, J. Kim, A.J. deMello and S.-I. Chang, "Integrated pneumatic micro-pumps for high-throughput dropletbased microfluidics", RSC Advances, 4, 2014, p. 20341.

[10] B. Li, L. Li, A. Guan, Q. Dong, K. Ruan, R. Hu and Z. Li, "A smartphone controlled handheld microfluidic liquid handling system", Lab Chip, 14, 2014, p. 4085-92.

[11] K.W. Oh, K. Lee, B. Ahn and E.P. Furlani, "Design of pressuredriven microfluidic networks using electric circuit analogy", Lab Chip, 12, 2012, p. 515-45. 